University of Nebraska - Lincoln

DigitalCommons@University of Nebraska - Lincoln

July 2001

\title{
Grain-boundary micromagnetism
}

\author{
Ralph Skomski \\ University of Nebraska-Lincoln, rskomski2@unl.edu
}

H. Zeng

University of Nebraska - Lincoln

David J. Sellmyer

University of Nebraska-Lincoln, dsellmyer@unl.edu

Follow this and additional works at: https://digitalcommons.unl.edu/physicssellmyer

Part of the Physics Commons

Skomski, Ralph; Zeng, H.; and Sellmyer, David J., "Grain-boundary micromagnetism" (2001). David Sellmyer Publications. 53.

https://digitalcommons.unl.edu/physicssellmyer/53

This Article is brought to you for free and open access by the Research Papers in Physics and Astronomy at DigitalCommons@University of Nebraska - Lincoln. It has been accepted for inclusion in David Sellmyer Publications by an authorized administrator of DigitalCommons@University of Nebraska - Lincoln. 


\title{
Grain-Boundary Micromagnetism
}

\author{
R. Skomski, H. Zeng, and D. J. Sellmyer
}

\begin{abstract}
Continuum and layer-resolved calculations are used to investigate the spin structure in the vicinity of grain boundaries. Reduced exchange in the grain-boundary region gives rise to a quasidiscontinuity of the magnetization and yields a perturbation which decays exponentially inside the grains. An effective intergranular exchange is obtained as a micromagnetically well-defined function of the grain-boundary exchange, and it is discussed how grain boundaries affect the hysteresis loops of nanostructures.
\end{abstract}

Index Terms-Anisotropy, grain boundaries, intergranular exchange, thin films.

\section{INTRODUCTION}

$\mathbf{T}$ HE micromagnetic spin structure at grain boundaries is of interest in the areas of permanent magnetism, magnetic recording, soft magnets, and spin-dependent transport. For example, inter-granular exchange tends to reduce the storage density of magnetic recording media but may be favorable in permanent magnets [1]-[7].

In permanent magnetism, the rationale is to expand the limited variety of suitable natural compounds [2], [4], [5], [8]. Based on the assumption of ideal interfaces between hard and soft grains, record energy products have been predicted in [2], but in practice it is difficult to realize the theoretical predictions [4], [5]. One reason is reduced exchange at grain boundaries [2], [5], [8], because a strong intergranular exchange coupling is required to synergize the advantages of the phases involved. On the other hand, the theoretical predictions are very well satisfied in single-crystalline $\mathrm{DyFe}{ }_{2} / \mathrm{YFe}_{2}$ multilayers, which are characterized by nearly ideal interfaces [9].

Ideal interfaces give rise to a variety of phenomena [8]. First, the effect of the interface is not limited to the adjacent atomic layers but extends well into the grain. In hard-magnetic grains, the penetration depth of the magnetic perturbation is given by the Bloch-wall width $\delta_{B}=(A / K)^{1 / 2}$ whereas in soft grains it extends throughout the grain. The effective coupling constant for two grains of arbitrary anisotropies $K_{s}$ and $K_{h}$

$$
J_{\mathrm{eff}}=2 L^{2} \frac{\sqrt{A K_{s} K_{h}}}{\sqrt{K_{h}}+\sqrt{K_{s}}}
$$

whereas $L$ is the grain size and $A$ is the exchange stiffness. For adjacent hard and soft grains, the coupling energy is delocalized and stored nearly entirely in the soft grain.

This paper focuses on the mesoscopic spin structure in the vicinity of nonideal grain boundaries. The basic idea is shown

Manuscript received October 13, 2000.

This work was supported by DOD, DOE, and CMRA.

The authors are with the Department of Physics and Astronomy and Center for Materials Research and Analysis, University of Nebraska, Lincoln, NE 68588 (e-mail: rskomski@unlserve.unl.edu).

Publisher Item Identifier S 0018-9464(01)07146-1.

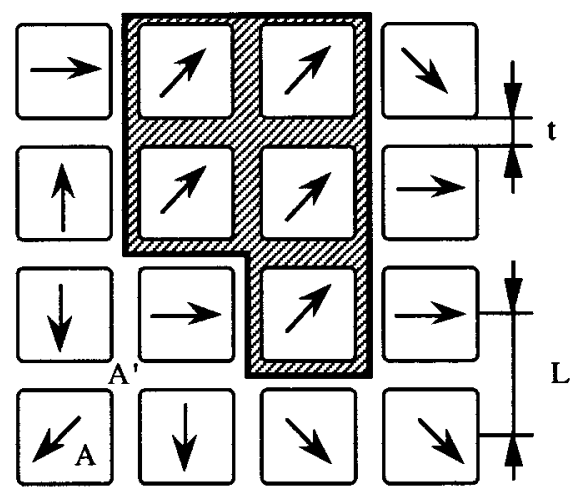

Fig. 1. Top view of a granular thin film (schematic). The arrows show the magnetization in the middle of each grain, and the no local easy axes may be aligned (oriented) or random.

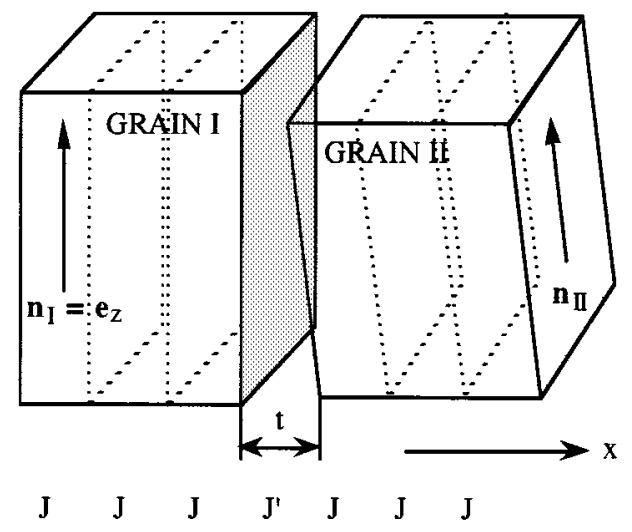

Fig. 2. Two neighboring grains. Far away from the boundary, the magnetization $\mathbf{M}$ is parallel to the grain easy axes $\mathbf{n}(\mathbf{r})$.

in Fig. 1: semihard or hard grains are embedded in an interaction matrix. The intergranular exchange, mediated by the matrix, leads to random-anisotropy correlations (shadowed area) whose size $R>L$ depends on the strength of the effective intergranular exchange.

The aim of this work is to determine the spin structure in the vicinity of the grain boundaries and to derive effective intergranular exchange constants from atomic parameters.

\section{INTERGRANULAR EXCHANGE}

The model is shown in Fig. 2: two adjacent but misaligned grains, characterized by the bulk exchange $J$, are separated by a boundary region of exchange $J^{\prime}$ and thickness $t$. (In the continuum approximation, $J$ and $J^{\prime}$ must be replaced by the respective exchange stiffnesses $A$ and $A^{\prime}$.) The anisotropy constant of the two grains is $K$, and their easy axes are given by the units vectors $\mathbf{n}_{\mathrm{I}}$ and $\mathbf{n}_{\mathrm{II}}$. To calculate the local magnetization $\mathbf{M}(\mathbf{r})$, which in general deviates from the easy-axis direction, one has 
to minimize the total magnetic energy $E$. The calculation is very similar to that used in [8].

In the continuum limit [2],

$$
E=\int\left(A(\mathbf{r}) \frac{(\nabla \mathbf{M})^{2}}{M_{s^{2}}}-K(\mathbf{r}) \frac{(\mathbf{n}(\mathbf{r}) \cdot \mathbf{M})^{2}}{M_{s^{2}}}\right) d V .
$$

Here we have ignored the influence of the magnetic field, because $A$ and $K$ are the leading energy contribution in hard magnets. Similarly, the spin structure at grain boundaries is only weakly perturbed by dipole interactions [10].

The constraint $|\mathbf{M}(\mathrm{r})|=M_{s}$ complicates the minimization of (1), but for small grain misalignment one can linearize the problem [10]:

$$
\mathbf{n}(\mathbf{r})=\left(1-a(\mathbf{r})^{2} / 2\right) \mathbf{e}_{z}+\mathbf{a}(\mathbf{r})
$$

where the easy-axis misorientation $|\mathbf{a}| \ll 1$, and

$$
\mathbf{M}(\mathbf{r})=M_{s}\left(1-m(\mathbf{r})^{2} / 2\right) \mathbf{e}_{z}+M_{s} \mathbf{m}(\mathbf{r})
$$

where the perpendicular magnetization component $m \ll 1$. Putting (2) into (3a) and (3b) and minimizing the energy with respect to $\mathbf{m}$ yields

$$
-\nabla(A(\mathbf{r}) \nabla \mathbf{m})+K(\mathbf{r}) \mathbf{m}=K(\mathbf{r}) \mathbf{a}(\mathbf{r}) .
$$

Since $A$ is discontinuous, we must use the boundary conditions [2] $\left.(A(x) \partial \mathbf{m} / \partial \mathbf{x})\right|_{x o-e}=\left.(A(x) \partial \mathbf{m} / \partial \mathbf{x})\right|_{x o+e}$.

Solving (4) for $K(\mathbf{r})=0$ in the boundary region yields a magnetization contribution decaying exponentially inside the grains and a quasidiscontinuity (magnetization jump) in the grain-boundary region. The fraction $\Delta$ of the magnetization change realized in the grain boundary is

$$
\Delta=\frac{1}{1+\frac{2 A^{\prime}}{t \sqrt{A K}}} .
$$

For $t=0$, the quasidiscontinuity vanishes $(\Delta=0)$, whereas zero intergranular exchange $\left(A^{\prime}=0\right)$ yields $\Delta=1$.

The energy $E$ stored in the grain-boundary region is a measure for the exchange coupling between the grains. Dividing $E$ by the product $\mathbf{n}_{\mathrm{I}} \cdot \mathbf{n}_{\mathrm{II}}$, which describes the degree of grain misalignment, we obtain

$$
J_{\mathrm{eff}}=L^{2} \sqrt{A K} /\left(1+\frac{t \sqrt{A K}}{2 A^{\prime}}\right) .
$$

This equation is a grain-boundary analog to the hard-soft result (1).

The magnetic continuum approximation breaks down on an atomic length scale [11]. In a layer-resolved analysis, (4) must be replaced by

$$
J_{n, n+1}\left(m_{n}-m_{n+1}\right)+K t_{o} m_{n}=K t_{o} a_{n}
$$

where $t_{o}$ is the thickness of the atomic layers. However, layerresolved and continuum calculations yield very similar results. Fig. 3 shows a spin structure obtained by solving (7).

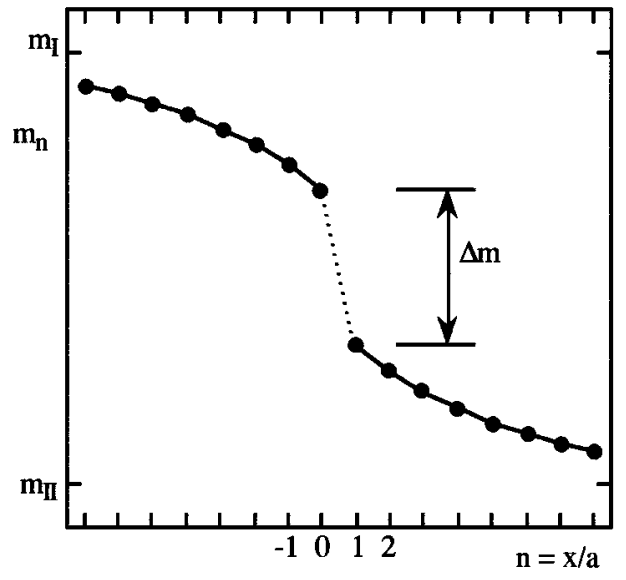

Fig. 3. Spin structure in the vicinity of the grain boundary. The jump $\Delta m$ amounts to a quasidiscontinuity of the magnetization at the grain boundary.

\section{DisCUSSION AND CONCLUSIONS}

The effect of the grain-boundary exchange on the magnetization depends on whether one considers magnetic equilibrium (domains) or nonequilibrium (hysteresis). For example, random-anisotropy magnets exhibit both ground-state domains (virgin curve at $H=0$ ) and "dynamic" domains during magnetization reversal (corresponding to the major hysteresis loop). However, the domain size during reversal may be much larger or much smaller than $d$-dimensional prediction

$$
R_{e q} \approx L\left(J_{\mathrm{eff}} / V K\right)^{2 /(4-d)}
$$

where $V$ is the grain volume. This equation, which describes the equilibrium of strongly interacting grains, is obtained by combining well-known random-anisotropy scaling relations with the $J_{\text {eff }}$ calculations presented in this work.

Another case is granular thin films characterized by a common easy-axis direction and a switching-field distribution. In this case, which is of some relevance in magnetic recording, there are no ground-state random-anisotropy domains $\left(R_{e q}=\infty\right)$, but micromagnetic localization [12] leads to the formation of domains during reversal.

It is well-known that intergranular exchange affects the hysteretic behavior of nanomagnets and leads, for example, to remanence enhancement. For grain sizes larger than $\delta_{B}$ one can show that sharp interfaces yield an enhancement $\Delta M / M_{s}$ scaling as $\delta_{B} / L$, whereas the smooth boundaries considered in [10] yield $\Delta M / M_{s} \sim \delta_{B}^{2} / L^{2}$.

In conclusion, we have investigated the effect of reduced interatomic exchange at grain-boundaries. Both the continuum and layer-resolved calculations, which can be considered as analytic complements to full-scale micromagnetic simulations [13], yield a quasidiscontinuity of the local magnetization. Other results are the derivation of a micromagnetically well-defined effective intergranular exchange constant $J_{\text {eff }}$ and scaling relations for the remanence enhancement.

\section{REFERENCES}

[1] G. C. Hadjipanayis, L. Withanawasam, and R. F. Krause, "Nanocomposite $\mathrm{Nd}_{2} \mathrm{Fe}_{14} \mathrm{~B} / \mathrm{alpha}-\mathrm{Fe}$ permanent magnets," IEEE Trans. Magn., vol. 31, pp. 3596-3601, 1995. 
[2] R. Skomski and J. M. D. Coey, "Giant energy product in nanostructured two-phase magnets," Phys. Rev. B, vol. 48, pp. 15 812-15 816, 1993.

[3] H. A. Davies, J. F. Liu, and G. Mendoza, "Recent progress in the study of remanence-enhanced nanocrystalline rare earth-iron-boron alloys," in Proc. 9th Int. Symp. REM, vol. 2, F. P. Missell et al., Eds.. Singapore, 1996, pp. 251-267.

[4] A. Al-Omari and D. J. Sellmyer, "Magnetic properties of nanostructured CoSm/FeCo films," Phys. Rev. B, vol. 52, pp. 3441-3447, 1995.

[5] J. P. Liu, C. P. Luo, Y. Liu, and D. J. Sellmyer, "High energy products in rapidly annealed nanoscale Fe/Pt multilayers," Appl. Phys. Lett., vol. 72, pp. 483-485, 1998.

[6] D. J. Sellmyer, M. Yu, R. A. Thomas, Y. Liu, and R. D. Kirby, "Nanoscale design of films for extremely high density magnetic recording," Phys. Low-Dim. Struct. 1-2, pp. 155-165, 1998.

[7] J. F. Löffler, H. B. Braun, and W. Wagner, "Magnetic correlations in nanostructured metals and an extended random-anisotropy model," $J$ Appl. Phys., vol. 85, pp. 5187-5189, 1999.

[8] R. Skomski, J. P. Liu, J. M. Meldrim, and D. J. Sellmyer, "Intergranular exchange in magnetic nanostructures," in Proc. 10th Int. Symp. REM, L. Schultz and K.-H. Müller, Eds. Frankfurt M.: Werkstoff informationsgesellschaft, 1998, pp. 277-286.
[9] Sawitzki, G. J. Bowden, P. A. J. de Groot, B. D. Rainford, J.-M. L. Beaujour, R. C. C. Ward, and M. R. Wells, "Exchange springs in antiferromagnetically coupled $\mathrm{DyFe}_{2} / \mathrm{YFe}_{2}$ superlattices," Phys. Rev. B, vol. 62, pp. 5817-5821, 2000.

[10] R. Skomski, J.-P. Liu, and D. J. Sellmyer, "Permanent magnetism in exchange-coupled nanocomposites," in Mat. Res. Soc. Symp. Proc., vol. 562, 1999, pp. 309-320.

[11] H. R. Hilzinger and H. Kronmüller, "Spin configuration and intrinsic coercive field of narrow domain walls in $\mathrm{Co}_{5} \mathrm{R}$-compounds," Phys. Stat. Sol. (b), vol. 54, pp. 593-604, 1972.

[12] R. Skomski and J. M. D. Coey, Permanent Magnetism. Bristol and Philadelphia: Institute of Physics, 1999.

[13] T. Schrefl, J. Fidler, and H. Kronmüller, "Remanence and coercivity in isotropic nanocrystalline permanent magnets," Phys. Rev. B, vol. 49, pp. 6100-6110, 1994 